(c) American Dairy Science Association, 2005.

\title{
Letter to the Editor: Comments on a Recent Article by Burgos et al.
}

\author{
T. R. Shryock, ${ }^{1}$ J. L. Watts, ${ }^{2}$ and J. T. Gray ${ }^{3}$ \\ ${ }^{1}$ Elanco Animal Health, Greenfield, IN 46140 \\ ${ }^{2}$ Pfizer Animal Health, Kalamazoo, MI 49001 \\ ${ }^{3}$ University of Guelph, Ontario Veterinary College, Ontario, Canada N1G 2W1
}

The publication by Burgos et al. (2005) raises several key issues regarding the use of antimicrobial susceptibility testing that are crucial to the entire study. First, the authors indicate that the 4 antimicrobial agents tested, chloramphenicol, nalidixic acid, tetracycline, and penicillin, were selected due to their widespread use in dairy cattle. Chloramphenicol has been banned from use in food-producing animals in the United States since the early 1980s and nalidixic acid is not used therapeutically in dairy cattle. Penicillin is not particularly active, nor used therapeutically, against the gramnegative enteric bacilli that were tested in the study. Tetracycline is used on dairy farms and, of the $4 \mathrm{com}$ pounds tested, the only one of any clinical relevance for the isolates tested. Second, Journal of Dairy Science Instructions to Authors (http://jds.fass.org/cgi/data/88/ 1/DC1/1) specify that antimicrobial susceptibility testing should be performed using a reproducible, standardized method such as those described by the Clinical and Laboratory Sciences Institute (formerly National Committee for Clinical Laboratory Standards; www.clsi.org). An integral part of any test method is the incorporation of routine quality control testing. Third,

Received July 25, 2005.

Accepted August 11, 2005.

Corresponding author: J. L. Watts; e-mail: jeffrey.l.watts@ pfizer.com. interpretive criteria used to categorize isolates as susceptible or resistant are linked to both the test method and the clinical efficacy of the agent. In this paper, the authors used a nonstandard method for determining the minimum inhibitory concentrations (MIC) of isolates, appear not to have performed quality control testing, and used the interpretive criteria from another method to categorize isolates as susceptible or resistant. As such, none of the MIC data or its interpretation as it presently appears in the Burgos et al. (2005) article meets the Journal of Dairy Science criteria for acceptance.

The issues with this paper highlight several key points to consider when performing antimicrobial susceptibility testing. The methods used should be standardized and quality control performed routinely. If interpretive criteria are applied to categorize isolates as susceptible or resistant, the source and relevance of those criteria should be plainly stated in the manuscript. Clearly, readers of the journal should interpret the data presented in the Burgos et al. (2005) paper carefully and consider the methodological and interpretive errors before citing it elsewhere.

\section{REFERENCES}

Burgos, J. M., B. A. Ellington, and M. F. Varela. 2005. Presence of multidrug resistant enteric bacteria in dairy farm topsoil. J. Dairy Sci. 88:1391-1398. 